\title{
MIXED-NORM GENERALIZATIONS OF BERGMAN SPACES AND DUALITY
}

\author{
STEVE GADBOIS
}

(Communicated by Irwin Kra)

\begin{abstract}
Conditions sufficient for boundedness of the Bergman projection on certain "mixed-norm" spaces of functions on the unit ball of $\mathbf{C}^{N}$ are given, and this is used to identify the dual space of such mixed-norm spaces. Several representation theorems that follow from the duality are also given.
\end{abstract}

1. Introduction. The classical Bergman space $A^{p}$ on the unit ball $B=B_{N}$ in $\mathrm{C}^{N}$ is the set of functions $f \in H\left(B_{N}\right)$ satisfying

$$
\left(\int_{B}|f(z)|^{p} d m(z)\right)^{1 / p}<\infty .
$$

Here $0<p<\infty$, the space $\mathbf{C}^{N}$ is equipped with the usual inner product defined for $z=\left(z_{1}, \ldots, z_{N}\right)$ and $w=\left(w_{1}, \ldots, w_{N}\right)$ in $\mathbf{C}^{N}$ by $\langle z, w\rangle=\sum_{i=1}^{N} z_{i} \overline{w_{i}}$ and with the associated norm $|z|=\langle z, z\rangle^{1 / 2}, H\left(B_{N}\right)$ is the set of holomorphic functions on $B_{N}$, and $m$ is Lebesgue measure on $B_{N}$ normalized so that $m\left(B_{N}\right)=1$. Using "polar coordinates" (see $[6,1.4 .3]$ ), this integral may be written as

$$
\left(2 N \int_{I}\left(\int_{S}|f(r \tau)|^{p} d \sigma(\tau)\right) r^{2 N-1} d r\right)^{1 / p}
$$

where $I=[0,1), S=S_{N}=\partial B_{N}$, and $\sigma$ is the rotation invariant positive Borel measure on $S_{N}$ with $\sigma\left(S_{N}\right)=1$.

We study here the following weighted "mixed norm" generalizations of the Bergman spaces. If $0<p, q<\infty$ and if $\alpha>-1$, define

$$
A_{\alpha}^{p q}=\left\{f \in H(B) \mid\|f\|_{p, q, \alpha}<\infty\right\}
$$

where

$$
\|f\|_{p, q, \alpha} \equiv\left(\int_{I}\left(\int_{S}|f(r \tau)|^{p} d \sigma(\tau)\right)^{q / p}\left(1-r^{2}\right)^{\alpha} r^{2 N-1} d r\right)^{1 / q} .
$$

Note that when $q=p$ and $\alpha=0$, this is precisely the Bergman space. Also note that

$$
\|f\|_{p, q, \alpha}=\left(\int_{I}\left\|f_{r}\right\|_{L^{p}(S)}^{q}\left(1-r^{2}\right)^{\alpha} r^{2 N-1} d r\right)^{1 / q}
$$

Received by the editors June 16, 1988.

1980 Mathematics Subject Classification (1985 Revision). Primary 32A10, 32H10.

Key words and phrases. Mixed-norm, Bergman space, dual. 
where, for $0 \leq r<1, f_{r}$ is the function defined on $S$ by $f_{r}(\tau)=f(r \tau)$. Using this notation, we also define

$$
\begin{gathered}
\|f\|_{\infty, q, \alpha} \equiv\left(\int_{I}\left\|f_{r}\right\|_{L^{\infty}(S)}^{q}\left(1-r^{2}\right)^{\alpha} r^{2 N-1} d r\right)^{1 / q} \\
\|f\|_{p, \infty} \equiv \sup _{0 \leq r<1}\left\|f_{r}\right\|_{L^{p}(S)}, \quad \text { and } \\
\|f\|_{\infty, \infty} \equiv \sup _{0 \leq r<1}\left\|f_{r}\right\|_{L^{\infty}(S)},
\end{gathered}
$$

and denote the spaces of holomorphic functions for which these are finite by $A_{\alpha}^{\infty q}$, $A^{p \infty}$, and $A^{\infty \infty}$ respectively. (The space $A^{p \infty}$ is the classical Hardy space $H^{p}$.) The set of (equivalence classes of) measurable functions satisfying the integrability condition defining $A_{\alpha}^{p q}$ is denoted by $L_{\alpha}^{p q}$. It is easy to check that

$$
\|f+g\|_{p, q, \alpha}^{s} \leq\|f\|_{p, q, \alpha}^{s}+\|g\|_{p, q, \alpha}^{s}
$$

where $s=\min \{p, q, 1\}$; thus $\left(A_{\alpha}^{p q},\|\|_{p, q, \alpha}^{s}\right)$ and $\left(L_{\alpha}^{p q},\|\|_{p, q, \alpha}^{s}\right)$ are metric spaces, and are normed linear spaces if $1 \leq p, q \leq \infty$. The basic reference for mixed norm spaces (including, but not limited to, our spaces $L_{\alpha}^{p q}$ ) is Benedek and Panzone, [2].

Note that by two applications of Hölder's inequality, $L_{\alpha_{1}}^{p_{1} q_{1}} \subset L_{\alpha_{2}}^{p_{2} q_{2}}$ if either

or

$$
p_{2} \leq p_{1} \quad \text { and } q_{2}<q_{1} \text { and } \frac{\left(\alpha_{1}+1\right)}{q_{1}}<\frac{\left(\alpha_{2}+1\right)}{q_{2}}
$$

$$
p_{2} \leq p_{1} \quad \text { and } \quad q_{2}=q_{1} \quad \text { and } \quad \alpha_{1} \leq \alpha_{2} .
$$

In either case, the containment is proper (unless $p_{1}=p_{2}, q_{1}=q_{2}$, and $\alpha_{1}=\alpha_{2}$ ), since then there is some constant $s$ satisfying the inequality $N / p_{1}+\left(\alpha_{1}+1\right) / q_{1}<s$ $<N / p_{2}+\left(\alpha_{2}+1\right) / q_{2}$, and $f(z)=(1-\langle z, \varsigma\rangle)^{-s}$ (with $\varsigma \in S$ fixed) defines a function in $A_{\alpha_{2}}^{p_{2} q_{2}}$ not in $A_{\alpha_{1}}^{p_{1} q_{1}}$ :

$$
\begin{aligned}
\|f\|_{p, q, \alpha}^{q} & =\int_{I}\left(\int_{S}|1-\langle r \tau, \zeta\rangle|^{-s p} d \sigma(\tau)\right)^{q / p}\left(1-r^{2}\right)^{\alpha} r^{2 N-1} d r \\
& \sim \int_{I}\left((1-r)^{N-s p}\right)^{q / p}(1-r)^{\alpha} r^{2 N-1} d r
\end{aligned}
$$

by Proposition 1.4 .10 of [6], and this integral is finite if and only if $s<N / p$ $+(\alpha+1) / q$. (We write $a(x) \sim b(x)$ if there exist constants $c$ and $C$ such that $0<c<$ $a(x) / b(x)<C<\infty$ as $x$ ranges over some index set.)

A few of the basic properties of $A_{\alpha}^{p q}$ are put forth in section two. Section three deals with boundedness of the Bergman projection, and this result is used to identify the dual space of our mixed norm spaces in $\S 4$. After introducing the pseudohyperbolic metric in $\S 5, \S 6$ and $\S 7$ are concerned with representations of the mixed norms and of functions in the mixed norm spaces. These results are generalizations of work of Luecking in [4].

This work represents some of the results contained in the author's Ph.D. dissertation completed at Michigan State University under the direction of Professor William Sledd.

2. Basic properties of $A_{\alpha}^{p q}$. The completeness of $A_{\alpha}^{p q}$ is a consequence of the following growth condition. Our statements and proofs here will resemble those in [7]. 
Proposition 2.1. If $f \in A_{\alpha}^{p q}(0<p, q \leq \infty,-1<\alpha)$, then

$$
|f(z)| \leq C\|f\|_{p, q, \alpha}(1-|z|)^{-(N / p+(\alpha+1) / q)} \quad \text { for every } z \in B
$$

for some $C$ independent of $f$.

PROOF. First suppose $0<p<\infty$. Since $|f|^{p}$ is plurisubharmonic, for $0<r<$ $s<1$ and $\tau \in S$ we have

$$
\begin{aligned}
|f(r \tau)|^{p} & \leq \int_{S}|f(s \eta)|^{p} P((r / s) \tau, \eta) d \sigma(\eta) \\
& \leq 2^{N}(s-r)^{-N} \int_{S}|f(s \eta)|^{p} d \sigma(\eta),
\end{aligned}
$$

i.e.,

$$
|f(r \tau)|(s-r)^{N / p} \leq C\left\|f_{s}\right\|_{L^{p}(S)} .
$$

Here $P$ denotes the invariant Poisson kernel defined for $z \in B$ and $\varsigma \in S$ by

$$
P(z, \varsigma)=\left(\frac{\left(1-|z|^{2}\right)}{|1-\langle z, \varsigma\rangle|^{2}}\right)^{N}
$$

For basic facts concerning the invariant Poisson kernel, see [6, Section 3.3]. If $q=\infty$, the result follows from (2.1) immediately. If $0<q<\infty$, we then have

$$
|f(r \tau)|^{q} \int_{r}^{1}(s-r)^{N q / p}(1-s)^{\alpha} s^{2 N-1} d s \leq C\|f\|_{p, q, \alpha}^{q} .
$$

Letting $x=(s-r) /(1-r)$, for $1 / 2 \leq r<1$ it follows that

$$
\begin{aligned}
C\|f\|_{p, q, \alpha}^{q} & \geq|f(r \tau)|^{q}(1-r)^{(N / p+(\alpha+1) / q) q} \int_{0}^{1} x^{N q / p}(1-x)^{\alpha}[(1-r) x+r]^{2 N-1} d x \\
& \sim|f(r \tau)|^{q}(1-r)^{(N / p+(\alpha+1) / q) q} \int_{0}^{1} x^{N q / p}(1-x)^{\alpha} d x
\end{aligned}
$$

so the result follows. If $0 \leq r<1 / 2$, the result follows from the maximum modulus theorem.

Now suppose $p=\infty$. Then $|f(r \tau)| \leq\left\|f_{s}\right\|_{L^{\infty}(S)}$ for $0 \leq r \leq s<1$, and the result for $0<q \leq \infty$ is proven by the same procedures.

COROLlaRY 2.2. If $1 \leq p, q \leq \infty$ and $-1<\alpha$, then $A_{\alpha}^{p q}$ is a closed subspace of $L_{\alpha}^{p q}$, and is hence a Banach space.

ProOF. Suppose $f_{n} \rightarrow f$ in $L_{\alpha}^{p q}$ with $f_{n} \in A_{\alpha}^{p q}$. Then there is a subsequence $f_{n_{k}}$ with $f_{n_{k}} \rightarrow f$ pointwise a.e. [2, p. 304]. By Proposition 2.1, we also have that $f_{n}$ is uniformly Cauchy on compact subsets of $B$, so $f_{n}$ is uniformly convergent on compact subsets of $B$ to some $g$ since $L_{\alpha}^{p q}$ is complete [2, p. 304]. But $g$ is analytic $[6,1.1 .4]$ and $f=g$ a.e., so $g \in A_{\alpha}^{p q}$ and $f_{n} \rightarrow g$ in $A_{\alpha}^{p q}$.

PROPOSITION 2.3. If $0<p, q<\infty$, then $\lim _{r \rightarrow 1^{-}}\left\|f_{r}-f\right\|_{p, q, \alpha}=0$ for every $f \in A_{\alpha}^{p q}$.

This follows immediately from the dominated convergence theorem. (For details, see [7, Proposition 3.3].) So the functions analytic in a neighborhood of $B$ form a dense subset of $A_{\alpha}^{p q}$. 
3. Boundedness of the Bergman projection on $L_{\alpha}^{p q}$. Suppose $t>-1$. The Bergman kernel $K_{t}$ is defined by

$$
K_{t}(z, w)=\frac{\left(1-|w|^{2}\right)^{t}}{(1-\langle z, w\rangle)^{N+1+t}}
$$

for $z, w \in B_{N}$. Note that:

(a) for fixed $w \in B, K_{t}(\cdot, w) \in A_{\alpha}^{p q}$ and

(b) for fixed $z \in B, K_{t}(z, \cdot) \in L_{\alpha}^{p q}$ if $t q>-(\alpha+1)$. (But $K_{t}(z, \cdot)$ is not conjugate holomorphic unless $t=0$.)

Both observations follow because the denominator of $K_{t}$ is bounded above and below in $B$.

The Bergman projection $T_{t}$ is defined by

$$
T_{t} f(z)=\left(\begin{array}{c}
N+t \\
N
\end{array}\right) \int_{B} K_{t}(z, w) f(w) d m(w)
$$

for $z \in B_{N}$ and $f$ for which the integrands are in $L^{1}(d m)$. In general, the binomial coefficient $\left(\begin{array}{c}N+t \\ N\end{array}\right)$ is $\frac{\Gamma(N+t+1)}{\Gamma(N+1) \Gamma(t+1)}$. It is clear that, for fixed $t, T_{t} f$ is holomorphic when defined.

In this section, a condition on $t, p, q$, and $\alpha$ will be found which ensures that $T_{t}$ is bounded on $L_{\alpha}^{p q}$; there will be no dependence on $p$ other than $p \geq 1$. In [3], Forelli and Rudin showed that $T_{t}$ is bounded on $L^{p}(d m)(1 \leq p<\infty)$ if and only if $(t+1) p>1$. Then in [1] Békollé showed that $T_{t}$ is bounded on $L^{p}\left(d m_{\alpha}\right)$ $\left(1<p<\infty,-1<\alpha, d m_{\alpha}(z)=\left(1-|z|^{2}\right)^{\alpha} d m(z)\right)$ if and only if $(t+1) p>\alpha+1$. (He actually showed this for more general weights satisfying a " $B_{p}$ condition", a condition analogous to Muckenhoupt's $A_{p}$ condition introduced in [5].) An important tool here will be the following pair of facts due to Forelli and Rudin in [3, Proposition 2.7]:

$$
\int_{B}\left|K_{t}(z, w)\right|\left(1-|w|^{2}\right)^{-c} d m(w) \leq C\left(1-|z|^{2}\right)^{-c}
$$
for every $z \in B$ if $0<c<t+1$;

$$
\int_{B}\left|K_{t}(z, w)\right|\left(1-|z|^{2}\right)^{-c} d m(z) \leq C\left(1-|w|^{2}\right)^{-c}
$$

for every $w \in B$ if $0<c+t<t+1$.

THEOREM 3.1. T $T_{t}$ maps $L_{\alpha}^{p q}(1 \leq p \leq \infty, 1<q<\infty,-1<\alpha)$ boundedly into $A_{\alpha}^{p q}$ if $(t+1) q>\alpha+1$. Furthermore, $T_{t} f=f$ and $T_{t} \bar{f} \equiv \overline{f(0)}$ for every $f \in A_{\alpha}^{p q}$.

Proof. As noted in [6, Proposition 7.1.2], $T_{t} f=f$ and $T_{t} \bar{f} \equiv \overline{f(0)}$ are true for $f \in H^{\infty}(B)$, hence for $f \in A_{\alpha}^{p q}$ by density of $H^{\infty}(B)$ in $A_{\alpha}^{p q}$, once continuity is verified. 
If $1<p<\infty$, vector-valued integration and Hölder's inequality yield that

$$
\begin{aligned}
\left\|\left(T_{t} f\right)_{r}\right\|_{L^{p}(S)} & =C\left\|\int_{I} \int_{S} K_{t}(r \cdot, s \eta) f_{s}(\eta) d \sigma(\eta) s^{2 N-1} d s\right\|_{L^{p}(S)} \\
\leq & C \int_{I}\left\|\int_{S} K_{t}(r \cdot, s \eta) f_{s}(\eta) d \sigma(\eta)\right\|_{L^{p}(S)} s^{2 N-1} d s \\
= & C \int_{I}\left(\int_{S}\left|\int_{S} K_{t}(r \tau, s \eta) f_{s}(\eta) d \sigma(\eta)\right|^{p} d \sigma(\tau)\right)^{1 / p} s^{2 N-1} d s \\
\leq & C \int_{I}\left[\int_{S}\left(\int_{S}\left|K_{t}(r \tau, s \eta) \| f_{s}(\eta)\right|^{p} d \sigma(\eta)\right)\right. \\
& \left.\times\left(\int_{S}\left|K_{t}(r \tau, s \eta)\right| d \sigma(\eta)\right)^{p / p^{\prime}} d \sigma(\tau)\right]^{1 / p} s^{2 N-1} d s .
\end{aligned}
$$

(As usual, $p^{\prime}$ denotes the conjugate exponent of $p$.) But $\int_{S}\left|K_{t}(r \tau, s \eta)\right| d \sigma(\eta)$ is independent of $\tau$, so by Fubini's theorem, the expression above is less than

$$
\begin{gathered}
C \int_{I}\left[\left(\int_{S}\left|K_{t}(r \tau, s \eta)\right| d \sigma(\eta)\right)^{p / p^{\prime}+1}\left(\int_{S}\left|f_{s}(\eta)\right|^{p} d \sigma(\eta)\right)\right]^{1 / p} s^{2 N-1} d s \\
=C \int_{I} \int_{S}\left|K_{t}(r \tau, s \eta)\right| d \sigma(\eta)\left\|f_{s}\right\|_{L^{p}(S)} s^{2 N-1} d s .
\end{gathered}
$$

This estimate can also be verified in similar way if $p=1$ or $p=\infty$.

Using this estimate and Hölder's inequality, we have

$$
\begin{aligned}
\left\|T_{t} f\right\|_{p, q, \alpha}^{q}= & C \int_{I}\left\|\left(T_{t} f\right)_{r}\right\|_{L^{p}(S)}^{q}\left(1-r^{2}\right)^{\alpha} r^{2 N-1} d r \\
\leq & C \int_{I}\left[\int_{I} \int_{S}\left|K_{t}(r \tau, s \eta)\right| d \sigma(\eta)\left\|f_{s}\right\|_{L^{p}(S)} s^{2 N-1} d s\right]^{q}\left(1-r^{2}\right)^{\alpha} r^{2 N-1} d r \\
\leq & C \int_{I}\left(\int_{B}\left|K_{t}(r \tau, s \eta)\right|\left(1-s^{2}\right)^{-\delta q^{\prime}} d m(s \eta)\right)^{q / q^{\prime}} \\
& \times\left(\int_{B}\left|K_{t}(r \tau, s \eta)\right|\left(1-s^{2}\right)^{\delta q}\left\|f_{s}\right\|_{L^{p}(S)}^{q} d m(s \eta)\right)\left(1-r^{2}\right)^{\alpha} r^{2 N-1} d r,
\end{aligned}
$$

where $\delta$ will be chosen later. But

$$
\int_{B}\left|K_{t}(r \tau, s \eta)\right|\left(1-s^{2}\right)^{-\delta q^{\prime}} d m(s \eta) \leq C\left(1-r^{2}\right)^{-\delta q^{\prime}}
$$

by (3.1a), as long as $0<\delta q^{\prime}<t+1$. Using this and Fubini's theorem, we have

$$
\begin{aligned}
\left\|T_{t} f\right\|_{p, q, \alpha}^{q} \leq & C \int_{I}\left(\left(1-r^{2}\right)^{-\delta q^{\prime}}\right)^{q / q^{\prime}} \\
& \times\left(\int_{B}\left|K_{t}(r \tau, s \eta)\right|\left(1-s^{2}\right)^{\delta q}\left\|f_{s}\right\|_{L^{p}(S)}^{q} d m(s \eta)\right)\left(1-r^{2}\right)^{\alpha} r^{2 N-1} d r \\
= & C \int_{I}\left\|f_{s}\right\|_{L^{p}(S)}^{q}\left(1-s^{2}\right)^{\delta q} \\
& \quad \times \int_{I} \int_{S}\left|K_{t}(r \tau, s \eta)\right| d \sigma(\eta)\left(1-r^{2}\right)^{\alpha-\delta q} r^{2 N-1} d r s^{2 N-1} d s .
\end{aligned}
$$


But

$$
\begin{gathered}
\int_{I} \int_{S}\left|K_{t}(r \tau, s \eta)\right| d \sigma(\eta)\left(1-r^{2}\right)^{\alpha-\delta q} r^{2 N-1} d r \\
\quad=\int_{B}\left|K_{t}(r \tau, s \eta)\right|\left(1-r^{2}\right)^{-(-\alpha+\delta q)} d m(r \tau) \\
\quad \leq C\left(1-s^{2}\right)^{-(-\alpha+\delta q)}=C\left(1-s^{2}\right)^{\alpha-\delta q}
\end{gathered}
$$

by (3.1b), as long as $0<-\alpha+\delta q+t<t+1$. So

$$
\left\|T_{t} f\right\|_{p, q, \alpha}^{q} \leq C \int_{I}\left\|f_{s}\right\|_{L^{p}(S)}^{q}\left(1-s^{2}\right)^{\alpha} s^{2 N-1} d s=C\|f\|_{p, q, \alpha}^{q} .
$$

To choose suitable $t$, note that there exists $\delta$ satisfying $0<\delta q^{\prime}<t+1$ and $0<-\alpha+\delta q+t<t+1$ if and only if

$$
(t+1) q>\alpha+1
$$

As in [3, p. 594], we immediately get the following.

COROllaRY 3.2. For $1 \leq p<\infty, 1<q<\infty$, and $-1<\alpha$, we have $\|f\|_{p, q, \alpha} \leq C\|\operatorname{Re} f\|_{p, q, \alpha}$ for every $f \in H(B)$ with $f(0)=0$.

Proof. Choose $t>(\alpha+1) / q-1$. Let $u=\operatorname{Re} f$ and fix $0<r, s<1$. Then $f_{s}=T_{t}\left(f_{s}\right)=T_{t}\left(f_{s}+\overline{f_{s}}\right)=2 T_{t}\left(u_{s}\right)$, so

$$
\int_{S}\left|f_{s}(r \tau)\right|^{p} d \sigma(\tau)=2^{p} \int_{S}\left|T_{t} u_{s}(r \tau)\right|^{p} d \sigma(\tau)
$$

Thus

$$
\begin{aligned}
\left\|f_{s}\right\|_{p, q, \alpha}^{q} & =\int_{I}\left(\int_{S}\left|f_{s}(r \tau)\right|^{p} d \sigma(\tau)\right)^{q / p}\left(1-r^{2}\right)^{\alpha} r^{2 N-1} d r \\
& =2^{q} \int_{I}\left(\int_{S}\left|T_{t} u_{s}(r \tau)\right|^{p} d \sigma(\tau)\right)^{q / p}\left(1-r^{2}\right)^{\alpha} r^{2 N-1} d r \\
& =2^{q}\left\|T_{t} u_{s}\right\|_{p, q, \alpha}^{q} \leq 2^{q} A^{q}\left\|u_{s}\right\|_{p, q, \alpha}^{q},
\end{aligned}
$$

and the result follows upon letting $s \rightarrow 1$.

4. Representation of the dual space of $A_{\alpha}^{p q}$. Representation of the dual space of $A_{\alpha}^{p q}$ will follow from boundedness of the Bergman projection (Theorem 3.1). The case $N=1, \alpha=0$ was handled by Shapiro in [7, Corollary 3.6]. In [4, Theorem 2.1], Luecking identified the dual space of $A_{\alpha}^{p}$ using the boundedness of the Bergman projection on $A_{\alpha}^{p}$ and $A_{\alpha\left(1-p^{\prime}\right)}^{p^{\prime}}$.

Given any fixed $g \in A_{\alpha\left(1-q^{\prime}\right)}^{p^{\prime} q^{\prime}}$, define the linear functional $L_{g}$ on $A_{\alpha}^{p q}$ by

$$
L_{g} f=\langle f, g\rangle=\int_{B} f \bar{g} d m
$$

for $f \in A_{\alpha}^{p q}$. By two applications of Hölder's inequality,

$$
|\langle f, g\rangle| \leq\|f\|_{p, q, \alpha}\|g\|_{p^{\prime}, q^{\prime}, \alpha\left(1-q^{\prime}\right)} .
$$


THEOREM 4.1. Suppose that $1<p<\infty, \max \{1, \alpha+1\}<q<\infty$, and $-1<\alpha$. Then the map taking $g$ to $L_{g}$ is a linear homeomorphism of $A_{\alpha\left(1-q^{\prime}\right)}^{p^{\prime} q^{\prime}}$ onto the dual space of $A_{\alpha}^{p q}$.

ProOF. As noted above, any $g \in A_{\alpha\left(1-q^{\prime}\right)}^{p^{\prime} q^{\prime}}$ defines a bounded linear functional $L_{g}$ on $A_{\alpha}^{p q}$, with $\left\|L_{g}\right\| \leq\|g\|_{p^{\prime}, q^{\prime}, \alpha\left(1-q^{\prime}\right)}$.

Now take any $L$ in the dual space $\left(A_{\alpha}^{p q}\right)^{*}$. Extend to $L \in\left(L_{\alpha}^{p q}\right)^{*}$ by the HahnBanach theorem. Write $\omega(r)=\left(1-r^{2}\right)^{\alpha}$, and note that $j \in L^{p q}$ if and only if $j \omega^{-1 / q} \in L_{\alpha}^{p q}$. Define the functional $\Lambda \in\left(L^{p q}\right)^{*}$ by $\Lambda j=L\left(j \omega^{-1 / q}\right)$; then there exists some function $k \in L^{p^{\prime} q^{\prime}}$ such that $\Lambda j=\int_{B} j k d m$. (See [2, Theorem 3.1] for the generalized representation theorem.) Let $h=\overline{k \omega^{1 / q}}$. We have $h \in L_{\alpha\left(1-q^{\prime}\right)}^{p^{\prime} q^{\prime}}$ and $L f=\langle f, h\rangle$ for all $f \in L_{\alpha}^{p q}$. Since $\overline{K_{0}(z, w)}=K_{0}(w, z)$, Fubini's theorem implies that

$$
\left\langle T_{0} f_{1}, f_{2}\right\rangle=\left\langle f_{1}, T_{0} f_{2}\right\rangle \text { for } f_{1} \in L_{\alpha}^{p q} \cap L^{2} \text { and } f_{2} \in L_{\alpha\left(1-q^{\prime}\right)}^{p^{\prime} q^{\prime}} \cap L^{2} .
$$

(To justify the application of Fubini's theorem, note that $T_{0} f_{1}$ and $T_{0} f_{2}$ are in $L^{2}$ since $f_{1}$ and $f_{2}$ are, either by Theorem 3.1 or by Békollé's result.) Now, $q>\alpha+1$ and $q^{\prime}>\alpha\left(1-q^{\prime}\right)+1$, so by Theorem 3.1, $T_{0}$ is bounded on $L_{\alpha}^{p q}$ and $L_{\alpha\left(1-q^{\prime}\right)}^{p^{\prime} q^{\prime}}$. By continuity of $T_{0}$ and density of the respective spaces [2, p. 308], (4.1) is also true for $f_{1} \in L_{\alpha}^{p q}$ and $f_{2} \in L_{\alpha\left(1-q^{\prime}\right)}^{p^{\prime} q^{\prime}}$. Let $g=T_{0} h$. So $g \in A_{\alpha\left(1-q^{\prime}\right)}^{p^{\prime} q^{\prime}}$, and for $f \in A_{\alpha}^{p q}$ we have $L f=\langle f, h\rangle=\left\langle T_{0} f, h\right\rangle=\left\langle f, T_{0} h\right\rangle=\langle f, g\rangle$, i.e., $L=L_{g}$.

If $g \in A_{\alpha\left(1-q^{\prime}\right)}^{p^{\prime} q^{\prime}}$ defines the zero functional, then since $\overline{K_{0}(z, \cdot)} \in A_{\alpha}^{p q}$ for any fixed $z \in B$, we have $0=\left\langle\overline{K_{0}(z, \cdot)}, g\right\rangle=\overline{T_{0} g(z)}=\overline{g(z)}$, i.e., $g \equiv 0$. So the map taking $g$ to $L_{g}$ is a one-to-one, continuous, linear transformation of $A_{\alpha\left(1-q^{\prime}\right)}^{p^{\prime} q^{\prime}}$ onto $\left(A_{\alpha}^{p q}\right)^{*}$. By the open mapping theorem, the map is actually a linear homeomorphism.

One can use other (i.e., weighted) duality pairings (and other kernels) to get other representations of $\left(A_{\alpha}^{p q}\right)^{*}$.

5. The pseudohyperbolic "metric". The pseudohyperbolic "metric" $\rho$ is defined on $B_{N}$ by $\rho(z, w)=\left|\Phi_{w}(z)\right|$ where $\Phi_{w}$ is the automorphism of $B_{N}$ given for $w \neq 0$ by

$$
\Phi_{w}(z)=\frac{w-\frac{\langle z, w\rangle}{\langle w, w\rangle} w-\left(1-|w|^{2}\right)^{1 / 2}\left(z-\frac{\langle z, w\rangle}{\langle w, w\rangle} w\right)}{1-\langle z, w\rangle}
$$

and for $w=0$ by $\Phi_{0}(z)=-z$. The corresponding "balls" are

$$
E(w, \delta)=\Phi_{w}^{-1}\left(\delta B_{N}\right)=\left\{z \in B_{N} \mid \rho(z, w)<\delta\right\}
$$

for $w \in B_{N}$ and $0<\delta<1$. Note that $m(E(w, \delta)) \sim \delta^{2 N}(1-|w|)^{N+1}$; see $[6,2.2 .7]$.

We will have need of the following.

LEMMA 5.1. Fix $0<r<1$ and $0<\delta$ small. Then

$$
\frac{r-\delta}{1-r \delta}<|z|<\frac{r+\delta}{1+r \delta} \quad \text { for every } z \in E(r, \delta) \text {. }
$$

(Here $E(r, \delta)$ means $E(w, \delta)$ with $\left.w=(r, 0, \ldots, 0) \in B_{N}.\right)$ 
ProOF. Write $z=\left(z_{1}, z_{2}, \ldots, z_{N}\right)=\left(z_{1}, z^{\prime}\right)$ and suppose $z \in E(r, \delta)$. Then

$$
\delta^{2}\left|1-r z_{1}\right|^{2}>\left|r-z_{1}\right|^{2}+\left(1-r^{2}\right)\left|z^{\prime}\right|^{2}
$$

i.e.,

$$
2 r\left(1-\delta^{2}\right) \operatorname{Re}\left(z_{1}\right)>\left(r^{2}-\delta^{2}\right)+\left(1-\delta^{2} r^{2}\right)\left|z_{1}\right|^{2}+\left(1-r^{2}\right)\left|z^{\prime}\right| .
$$

Now, $4 r \operatorname{Re}\left(z_{1}\right)+r^{2}\left|z^{\prime}\right|^{2}<r^{2}+4 r\left|z_{1}\right|-r^{2}\left|z_{1}\right|^{2} \leq 4 r<4$, so

$$
\begin{aligned}
\left(r^{2}-\right. & \left.\delta^{2}\right)+\left(1-\delta^{2} r^{2}\right)|z|^{2} \\
= & {\left[\left(r^{2}-\delta^{2}\right)+\left(1-\delta^{2} r^{2}\right)\left|z_{1}\right|^{2}+\left(1-r^{2}\right)\left|z^{\prime}\right|^{2}\right]+r^{2}\left(1-\delta^{2}\right)\left|z^{\prime}\right|^{2} } \\
& <2 r\left(1-\delta^{2}\right)\left(\operatorname{Re}\left(z_{1}\right)+r\left|z^{\prime}\right|^{2} / 2\right) \\
\leq & 2 r\left(1-\delta^{2}\right)\left(\left|z_{1}\right|^{2}+r\left|z^{\prime}\right|^{2} \operatorname{Re}\left(z_{1}\right)+r^{2}\left|z^{\prime}\right|^{4} / 4\right)^{1 / 2} \\
& <2 r\left(1-\delta^{2}\right)\left(\left|z_{1}\right|^{2}+\left|z^{\prime}\right|^{2}\right)^{1 / 2}=2 r\left(1-\delta^{2}\right)|z| .
\end{aligned}
$$

Hence $\left(|z|, 0^{\prime}\right) \in E(r, \delta)$, and $(r-\delta) /(1-r \delta)<|z|<(r+\delta) /(1+r \delta)$.

6. A norm-representation theorem. In [4, Theorem 5.1], Luecking shows that

$$
\|f\|_{H^{p}} \sim\left(\sup _{m} \sum_{k=1}^{k_{m}}\left|f\left(a_{m k}\right)\right|^{p}\left(1-r_{m}\right)^{N}\right)^{1 / p} \quad \text { for all } f \in H^{p}
$$

where $0 \leq r_{0}<r_{1}<\cdots \rightarrow 1$ and $\left\{a_{m k}\right\}$ satisfies

(1) $\left|a_{m k}\right|=r_{m}$ for each $m=0,1,2, \ldots$ and each $k=1,2, \ldots, k_{m}$,

(2) $r_{m} S_{N} \subset \bigcup_{k} E\left(a_{m k}, \delta\right)$ for each $m$ for some $\delta=\delta(p)$ sufficiently small, and

(3) $E\left(a_{m k}, \varepsilon\right) \cap E\left(a_{m k^{\prime}}, \varepsilon\right)=\varnothing$ for each $m$ and each $k \neq k^{\prime}$ for some $0<\varepsilon(p)<\delta$. Such a set of points $\left\{a_{m k}\right\}$ will be called an $\varepsilon-\delta$ lattice.

A close analysis of Luecking's proof yields the following.

THEOREM 6.1. Fix $0<p<\infty, 0<q<\infty$, and $-1<\alpha$. Let $r_{m}=1-2^{-m}$ for $m=0,1,2, \ldots$ and suppose $\left\{a_{m k}\right\}$ is an $\varepsilon-\delta$ lattice for $\delta=\delta(p, q, \alpha)$ sufficiently small. Then

$$
\|f\|_{p, q, \alpha} \sim\left(\sum_{m=0}^{\infty}\left(\sum_{k=1}^{k_{m}}\left|f\left(a_{m k}\right)\right|^{p} 2^{-m N}\right)^{q / p} 2^{-m(\alpha+1)}\right)^{1 / q} \text { for every } f \in A_{\alpha}^{p q} .
$$

PROOF. Let $r_{m \varepsilon}=\left(r_{m}+\varepsilon\right) /\left(1+r_{m} \varepsilon\right), E_{m k}=E\left(a_{m k}, \varepsilon\right), A_{m}=\bigcup_{k} E_{m k}$, and $I_{m \varepsilon}=\left[\left(r_{m}-\varepsilon\right) /\left(1-r_{m} \varepsilon\right),\left(r_{m}+\varepsilon\right) /\left(1+r_{m} \varepsilon\right)\right)$. Then by plurisubharmonicity of $|f|^{p}$, the separation property (3), and Lemma 5.1,

$$
\begin{aligned}
\sum_{k} \mid & \left.f\left(a_{m k}\right)\right|^{p} 2^{-m N} \leq 2^{-m N}\left(\sum_{k} C \frac{\int_{E_{m k}}|f|^{p} d m}{m\left(E_{m k}\right)}\right) \\
& \leq C 2^{-m N} 2^{m(N+1)} \sum_{k} \int_{E_{m k}}|f|^{p} d m=C 2^{m} \int_{A_{m}}|f|^{p} d m \\
& \leq C 2^{m} \int_{I_{m \varepsilon}}\left(\int_{S}|f(r \tau)|^{p} d \sigma(\tau)\right) r^{2 N-1} d r \\
& \leq C 2^{m}\left(\int_{I_{m \varepsilon}} r^{2 N-1} d r\right)\left\|f_{r_{m \varepsilon}}\right\|_{L^{p}(S)}^{p} \leq C\left\|f_{r_{m \varepsilon}}\right\|_{L^{p}(S)}^{p} .
\end{aligned}
$$


Since we may assume that $\varepsilon<1 / 3$ (at the cost of increasing the constant $C$ ), we have $r_{m \varepsilon}<\left(r_{m}+1\right) / 2=r_{m+1}$, and thus

$$
\begin{gathered}
\sum_{m}\left(\sum_{k}\left|f\left(a_{m k}\right)\right|^{p} 2^{-m N}\right)^{q / p} 2^{-m(\alpha+1)} \leq C \sum_{m}\left\|f_{r_{m+1}}\right\|_{L^{p}(S)}^{q} 2^{-m(\alpha+1)} \\
\leq C \int_{I}\left\|f_{r}\right\|_{L^{p}(S)}^{q}(1-r)^{\alpha} r^{2 N-1} d r=C\|f\|_{p, q, \alpha}^{q} .
\end{gathered}
$$

In the other direction, Luecking uses a change of variables, Fubini's theorem, and the "denseness" property (2) and actually shows that

so

$$
c\left\|f_{r_{m}}\right\|_{L^{p}(S)}^{p} \leq C \delta^{p}\left\|f_{r_{m \varepsilon}}\right\|_{L^{p}(S)}^{p}+\sum_{k}\left|f\left(a_{m k}\right)\right|^{p} 2^{-m N}
$$

$$
\begin{aligned}
c\|f\|_{p, q, \alpha}^{q} & \leq \sum_{m}\left(C \delta^{p}\left\|f_{r_{m+1}}\right\|_{L^{p}(S)}^{p}+\sum_{k}\left|f\left(a_{m k}\right)\right|^{p} 2^{-m N}\right)^{q / p} 2^{-m(\alpha+1)} \\
& \leq C^{\prime} \sum_{m}\left(C \delta^{q}\left\|f_{r_{m+1}}\right\|_{L^{p}(S)}^{q}+\left(\sum_{k}\left|f\left(a_{m k}\right)\right|^{p} 2^{-m N}\right)^{q / p}\right) 2^{-m(\alpha+1)}
\end{aligned}
$$

where $C^{\prime}=1$ if $q \leq p$ and $C^{\prime}=2^{q / p-1}$ if $p<q$. This is less than

$$
\begin{gathered}
C \delta^{q} \sum_{m}\left\|f_{r_{m+1}}\right\|_{L^{p}(S)}^{q} 2^{-m(\alpha+1)}+C \sum_{m}\left(\sum_{k}\left|f\left(a_{m k}\right)\right|^{p} 2^{-m N}\right)^{q / p} 2^{-m(\alpha+1)} \\
\leq C \delta^{q}\|f\|_{p, q, \alpha}^{q}+C \sum_{m}\left(\sum_{k}\left|f\left(a_{m k}\right)\right|^{p} 2^{-m N}\right)^{q / p} 2^{-m(\alpha+1)}
\end{gathered}
$$

and the result follows for $\delta$ sufficiently small.

As a consequence of this theorem, no such $\varepsilon-\delta$ lattice can be a subset of the zero set of an $A_{\alpha}^{p q}$ function not identically zero.

7. Representation of $A_{\alpha}^{p q}$ functions. The duality result and the equivalence of norms result (Theorems 3.1 and 6.1) can be used to obtain a representation of $A_{\alpha}^{p q}$ functions as sums of kernel functions. This generalizes Luecking's Corollary 4.4 in [4].

If $v$ is a weight function on $\{0,1,2, \ldots\}$, we write $c=\left\{c_{m k}\right\}_{m, k} \in l_{v}^{p q}$ if

$$
\left(\sum_{m=0}^{\infty}\left(\sum_{k=1}^{\infty}\left|c_{m k}\right|^{p}\right)^{q / p} v_{m}\right)^{1 / q} \equiv\|c\|_{p, q, v}<\infty .
$$

THEOREM 7.1. Suppose $1<p<\infty, 1<q<\infty$, and $-1<\alpha$. Let $r_{m}=1-2^{-m}$ and $v_{m}=\left(1-r_{m}\right)^{1+N q^{\prime} / p^{\prime}+\alpha\left(1-q^{\prime}\right)} r_{m}^{2 N-1}$ for each $m=0,1,2, \ldots$, and suppose $\left\{a_{m k}\right\}$ is an $\varepsilon-\delta$ lattice for $\delta=\delta(p, q, \alpha)$ sufficiently small. Then every $f \in A_{\alpha}^{p q}$ is of the form

$$
f(z)=\sum_{m=0}^{\infty} \sum_{k=1}^{k_{m}} c_{m k} v_{m}\left(1-\left\langle z, a_{m k}\right\rangle\right)^{-N-1}
$$

for some $c \in l_{v}^{p q}$, and any $f$ of this form is in $A_{\alpha}^{p q}$. 
Note that no claim of uniqueness of $c \in l_{v}^{p q}$ is being made.

PROOF. As in the proof of Theorem 6.1,

$\|g\|_{p^{\prime}, q^{\prime}, \alpha\left(1-q^{\prime}\right)} \sim\left(\sum_{m}\left(\sum_{k}\left|g\left(a_{m k}\right)\right|^{p^{\prime}}\right)^{q^{\prime} / p^{\prime}}\left(1-r_{m}\right)^{1+N q^{\prime} / p^{\prime}+\alpha\left(1-q^{\prime}\right)} r_{m}^{2 N-1}\right)^{1 / q^{\prime}}$ i.e.,

$$
\|g\|_{p^{\prime}, q^{\prime}, \alpha\left(1-q^{\prime}\right)} \sim\left\|g\left(a_{m k}\right)\right\|_{p^{\prime}, q^{\prime}, v} .
$$

Thus the map $R: A_{\alpha\left(1-q^{\prime}\right)}^{p^{\prime} q^{\prime}} \rightarrow l_{v}^{p^{\prime} q^{\prime}}$ defined by $(R g)_{m k}=g\left(a_{m k}\right)$ is a linear isomorphism. Hence $R$ is one-to-one with closed range, and $R^{*}: l_{v}^{p q} \rightarrow A_{\alpha}^{p q}$ is onto. (Since $\max \left\{1, \alpha\left(1-q^{\prime}\right)+1\right\}<q^{\prime}<\infty$, we have $\left(A_{\alpha\left(1-q^{\prime}\right)}^{p^{\prime} q^{\prime}}\right)^{*} \sim A_{\alpha}^{p q}$ by Theorem 3.1, where the duality pairing does not involve a weight, and we have $\left(l_{v}^{p^{\prime} q^{\prime}}\right)^{*} \sim l_{v}^{p q}$ by Theorem 3.1 of $[2]$, where the duality pairing does involve the weight $v$.)

To identify $R^{*}$, take $g \in A_{\alpha\left(1-q^{\prime}\right)}^{p^{\prime} q^{\prime}}$ and $c \in l_{v}^{p q}$, supposing first that $c$ has only finitely many nonzero terms. Then

$$
\begin{aligned}
\int_{B}\left(R^{*} c\right) g d m & =\left\langle R^{*} c, g\right\rangle=\langle c, R g\rangle=\sum_{m}\left(\sum_{k} c_{m k} \overline{g\left(a_{m k}\right)}\right) v_{m} \\
& =\sum_{m} \sum_{k} c_{m k} v_{m} \overline{\int_{B} g(z)\left(1-\left\langle a_{m k}, z\right\rangle\right)^{-N-1} d m(z)} \\
& =\int_{B}\left(\sum_{m} \sum_{k} c_{m k} v_{m}\left(1-\left\langle z, a_{m k}\right\rangle\right)^{-N-1}\right) \overline{g(z)} d m(z),
\end{aligned}
$$

so $R^{*} c(z)=\sum_{m} \sum_{k} c_{m k} v_{m}\left(1-\left\langle z, a_{m k}\right\rangle\right)^{-N-1}$. To get the result for general $c \in l_{v}^{p q}$, use finite approximations to $c$ (for which the result was just verified), the continuity of $R^{*}$, and the fact that convergence in $A_{\alpha}^{p q}$ implies pointwise convergence.

\section{REFERENCES}

1. D. Békollé, Inégalitiés à poids pour le projecteur de Bergman dans la boule unité de $\mathbf{C}^{n}$, Studia Math. 71 (1982), 305-323.

2. A. Benedek and R. Panzone, The spaces $L^{p}$, with mixed norm, Duke Math. J. 28 (1961), 301-324.

3. F. Forelli and W. Rudin, Projections on spaces of holomorphic functions in balls, Indiana Univ. Math. J. 24 (1974), 593-602.

4. D. Luecking, Representation and duality in weighted spaces of analytic functions, Indiana Univ. Math. J. 34 (1985), 319-336.

5. B. Muckenhoupt, Weighted norm inequalities for the Hardy maximal function, Trans. Amer. Math. Soc. 165 (1972), 207-226.

6. W. Rudin, Function theory in the unit ball of $C^{n}$, Springer-Verlag, 1980.

7. J. Shapiro, Linear functionals on non-locally convex spaces, Ph.D. dissertation, University of Michigan, 1968.

Department of Mathematics, Rhodes College, 2000 North Parkway, MemPHIS, TENNESSEE 38112-1624 